\title{
ARBORIZAÇÃO URBANA EM JEQUITAÍ - MG: ATRIBUTOS FUNCIONAIS E DIVERSIDADE
}

\author{
URBAN TREES IN JEQUITAÍ-MG: FUNCTIONAL ATTRIBUTES AND DIVERSITY
}

Gislene Rodrigues dos Santos¹, Rúbia Santos Fonseca², Cristieli Barros Gonçalves¹

\section{RESUMO}

Objetivou-se caracterizar a arborização urbana quanto à diversidade, ecologia e interação com ambiente, por meio de inventário em todas praças (sete) e avenidas (quatro) da cidade de Jequitaí, Minas Gerais. Foi realizado o levantamento de todos os indivíduos com circunferência a $1,30 \mathrm{~m}$ do solo, maior ou igual a $15 \mathrm{~cm}$, incluindo troncos e estipes. O grupo de polinizadores foi definido por meio das síndromes de polinização e para a determinação do agente dispersor foram utilizadas as síndromes de dispersão. Foram avaliados 111 indivíduos, distribuídos em 16 famílias, 23 gêneros e 24 espécies. Em relação à origem das espécies, $37 \%$ são nativas ( $8 \%$ nativas de outras regiões do Brasil, $29 \%$ são nativas da região) e $63 \%$ são exóticas. A melitofilia se configura a síndrome de polinização mais frequente entre as espécies analisadas, enquanto a zoocoria foi o modo de dispersão mais ocorrente. A arborização da cidade de Jequitaí é composta em sua maioria por espécies exóticas. A arborização apresenta baixa diversidade, sendo Licania tomentosa a mais frequente. As espécies nativas da região como Cordia glabrata, Hymenaea courbaril e Genipa americana, se destacam por interagirem com a fauna e apresentarem bom estado de conservação.

Palavras-chave: Biodiversidade; Ecologia urbana; Ecossistema urbano; Floresta urbana.

\section{ABSTRACT}

The objective of this study was to characterize the urban afforestation in relation to diversity, ecology and interaction with the environment, throughout an inventory of all the squares (seven) and avenues (four) of the city of Jequitaí, in Minas Gerais. It was carried out a survey of all the individuals with circumference at $1.30 \mathrm{~m}$ of the soil, greater or equal to $15 \mathrm{~cm}$, including trees and palm trees. The group of pollinators was defined by the pollination syndromes and to determine the dispersal agent it was used the dispersal syndromes. We evaluated 111 individuals, distributed in 16 families, 23 genera and 24 species. Regarding the origin of the species, $37 \%$ are native ( $8 \%$ from other regions of Brazil, $29 \%$ are native to the region) and $63 \%$ are exotic. Melittophily constitutes the more frequent pollination syndrome among the analyzed species, whereas zoochory was the most frequent dispersal mode. The afforestation of the city of Jequita is constituted mostly by exotic species. The afforestation has low diversity, with Licania tomentosa being the most frequent species. The native species of the region such as Cordia glabrata, Hymenaea courbaril and Genipa americana, stand out for interacting with the fauna and receiving a good state of conservation.

Keywords: Biodiversity; Urban ecology; Urban ecosystem; Urban forest.

Recebido em 14.03.2019 e aceito em 24.05.2019

1.Engenheira Florestal formada, pela UFMG. Montes Claros/MG. Email: gislenerodrigues58@yahoo.com.br/ cristielligoncalves@gmail.com

2 Bióloga. Doutora em Botânica pela UFV. Montes Claros/MG. Email: rubiafonseca@hotmail.com 


\section{INTRODUÇÃO}

Nos últimos 60 anos, o mundo passou por grande intensificação no processo de urbanização, atualmente mais da metade da população vive em centros urbanos e a previsão é que a população urbana permaneça aumentando (UNITED NATIONS, 2015). As grandes alterações na cobertura da superfície do solo, pela substituição da vegetação natural, construção de edificações, e, consequentemente, impermeabilização do solo e aumento da irradiação de calor para a atmosfera, causam desconforto térmico e desequilíbrio ambiental, formando ilhas de calor (COSTA; PERES; SILVA, 2009).

A carência de planejamento urbano promove modificações indesejadas no ciclo hidrológico, pois altera a drenagem e aumenta a probabilidade de ocorrência de enchentes e deslizamentos, implicando em riscos à saúde e vida humana (BENINI; MENDIONDO, 2015). A vegetação remanescente nas cidades participa do ciclo hidrológico, colaborando para mitigação de enchentes e inundações (CADORIN; MELLO, 2011; GÓMEZ-BAGGETHUN; BARTON, 2013).

A arborização fornece serviços ecossistêmicos que são fundamentais para a qualidade de vida na cidade, amenizando os efeitos causados pela urbanização (MATOS; QUEIROZ, 2009). Ambientes com plantas são preferidos, pois elas reduzem a temperatura, abrandando assim os efeitos formados pelas ilhas de calor, protegem da poeira, ventos, reduzem ruídos, trazem beleza para a cidade e colaboram com a saúde mental (CADORIN; MELLO, 2011; GÓMEZ-BAGGETHUN; BARTON, 2013; MATOS; QUEIROZ, 2009).

É recomendado utilizar mais de uma espécie na arborização de vias públicas, pois irão funcionar como corredor ecológico urbano para a fauna e são fonte de recursos alimentares para polinizadores e dispersores presente nestes locais (BRUN; LINK; BRUN, 2007). Além disso, a diversidade de espécies na arborização é de suma importância, pois diminui os riscos de perdas da vegetação por ataques de insetos ou doenças (CEMIG, 2011).

Diante da importância ecológica e social da vegetação urbana, é fundamental conhecer as espécies que compõe a vegetação das cidades. Sendo assim, objetivou-se caracterizar a vegetação urbana quanto à sua diversidade, ecologia e interação com o ambiente, por meio de inventário total nas praças e avenidas da cidade de Jequitaí, Minas Gerais.

\section{MATERIAL E MÉTODOS}

\section{Área de estudo}

O Município de Jequitaí está situado na região norte do estado de Minas Gerais (coordenadas: $17^{\circ} 13^{\prime} 48.11^{\prime \prime S}$ e 44²6'15.99"O) (Figura 1). A área total do município é de 1.268,4 
$\mathrm{Km}^{2}$ e a população estimada de aproximadamente 7.597 habitantes. Em 2010 o IDMH (Índice de desenvolvimento humano municipal) foi 0,6643 (IBGE, 2017).

O município de Jequitaí está imerso no Domínio Cerrado. A cidade está localizada às margens do rio que recebe o mesmo nome da cidade. O rio Jequitaí é um importante afluente do Rio São Francisco. Em Jequitaí, diversos rios e córregos enfrentam problemas com a seca, principalmente em função do desmatamento e assoreamento destes cursos d'água. O clima da região se caracteriza como Aw, de acordo com a Classificação climática de Köppen-Geiger, Clima tropical com estação seca.

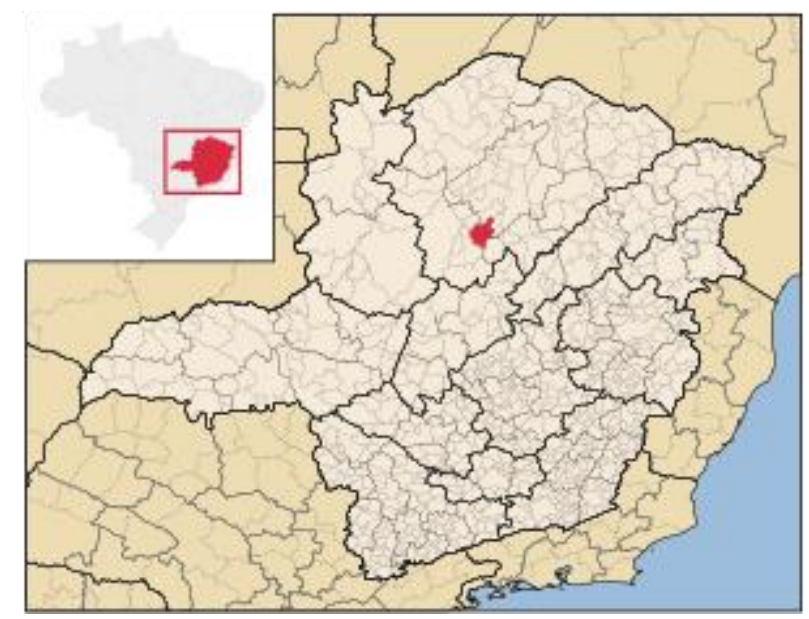

Figura 1. Localização da cidade de Jequitaí no norte de Minas Gerais

Figure 1. Location of the city of Jequitaí in the north of Minas Gerais

\section{Procedimentos metodológicos}

O inventário foi realizado entre janeiro e agosto de 2017, foram analisados todos os indivíduos com CAP (circunferência à altura do peito, a 1,30m do solo) maior ou igual a $15 \mathrm{~cm}$, incluindo troncos e estipes. A medição da altura dos indivíduos foi realizada utilizando a prancheta dendrométrica. $O$ inventário incluiu todas praças e avenidas da cidade. Foram avaliadas sete praças (Praça Coronel Daniel da Fonseca, Praça José Augusto de Oliveira, Praça José Bonifácio de Andradas, Praça Maria Alves, Praça Cristo Redentor, Praça Miguel da Malária, Praça do Locomóvel) e quatro avenidas (Avenida Sete de Setembro, Avenida dos Andradas, Avenida Estados Unidos, Avenida Espirito Santo) da cidade (Figura 2).

Para cada indivíduo foram anotadas as informações: nome popular (quando possível), altura, circunferência (os dados de circunferência foram transformados em diâmetro) e conflito com elementos estruturais pré-existentes (rede aérea de energia elétrica e calçadas). Para rede aérea de energia elétrica, foi considerado conflito quando a rede estava entre a copa ou em contato com a copa e para as calçadas quando havia rachaduras e elevações da calçada. A poda foi avaliada, sendo classificada em drástica, quando toda a copa estava suprimida e leve quando apenas alguns galhos foram eliminados. Além disso, os indivíduos foram divididos em 
categorias conforme seu estado geral (ótima, boa, regular e péssima), seguindo o proposto por Silva Filho et al. (2002).

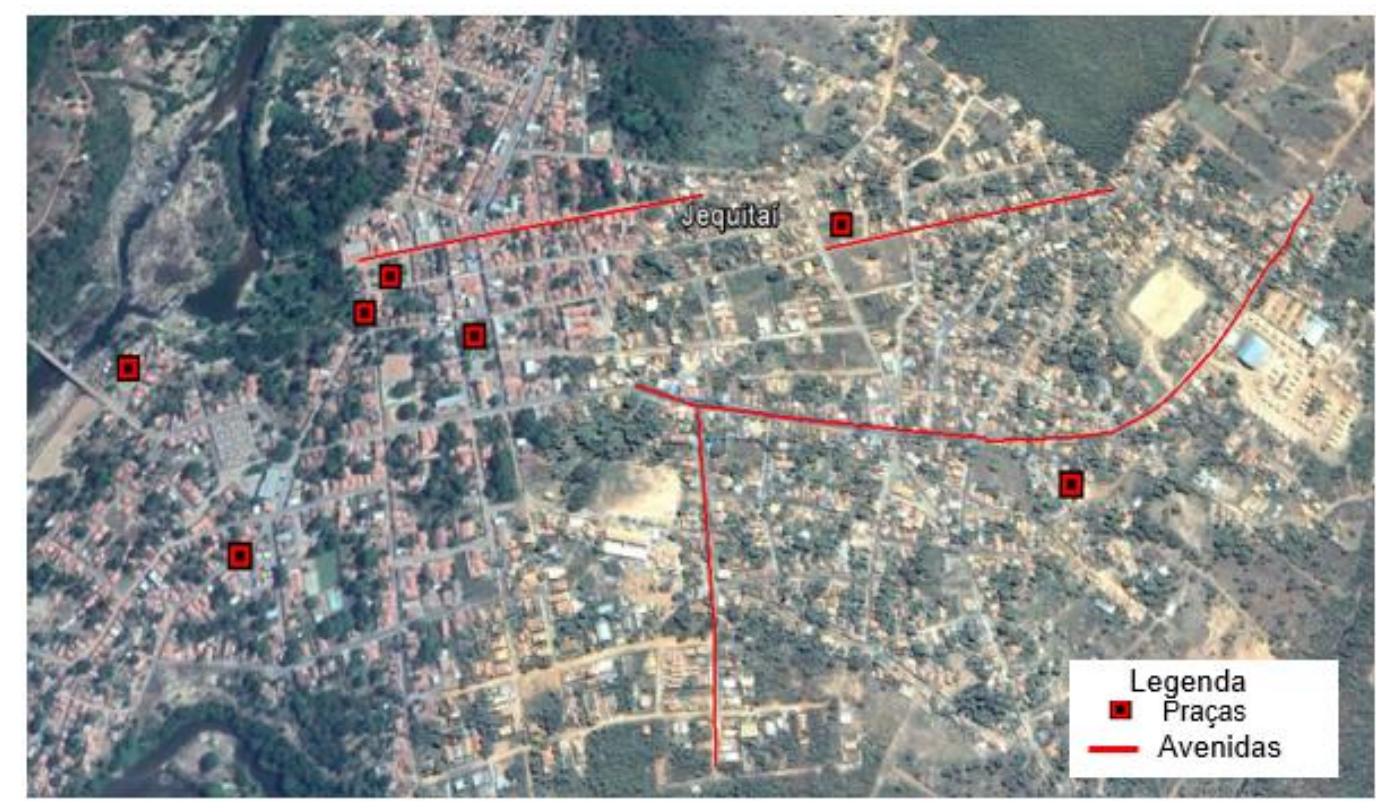

Fonte: Adaptado Google Earth (2018)

Figura 2. Praças e avenidas do município de Jequitaí no norte de Minas Gerais

Figure 2. Squares and avenues of the city of Jequitaí in the north of Minas Gerais

A origem das espécies foi determinada com base na Flora do Brasil (2019) e outras referências especializadas, segundo Pastore et al. (2013) foram classificadas em: nativa da região, nativa do Brasil e exóticas cultivadas (não sobrevivem sem o cultivo do homem e exótica naturalizadas (adaptam ao novo ambiente e passam a se reproduzir sem a intervenção direta do homem).

O grupo de polinizadores foi definido por meio das síndromes de polinização, para tanto a morfologia, biologia floral e recursos florais foram analisados e seguidos os critérios de Faegri e Pijl (1976). Para as espécies que não floresceram durante o estudo foi realizada consulta a material de herbário. As síndromes de polinização das flores foram divididas em cantarofilia (polinização por besouro), melitofilia (polinização por abelhas), entomofilia (polinização por insetos em geral), ornitofilia (polinização por aves), quiropterofilia (polinização por morcegos), esfingofilia ou falenofilia (polinização por mariposas) e anemofilia (polinização pelo vento).

Para a determinação do agente dispersor foram utilizadas as síndromes de dispersão (sensu VAN DER PIJL, 1982), cujos diásporos foram classificados em: zoocóricos, anemocóricos e autocóricos. Para as espécies que não frutificaram durante o período do estudo, foram consultadas exsicatas em herbários.

Foi determinada a Frequência Absoluta (FA), isto é o número de indivíduos por espécie, e a Frequência Relativa (FR), a proporção de indivíduos de cada espécie na arborização urbana. 
Para a análise da diversidade foi usado o índice de Shannon ( $\left.H^{\prime}\right)$ e de equabilidade (J') (MAGURRAN, 2011).

\section{RESULTADOS E DISCUSSÃO}

Foram avaliados 111 indivíduos, distribuídos em 16 famílias, 23 gêneros e 24 espécies. A família com maior frequência foi Chrysobalanaceae, com 37 indivíduos de uma espécie, Licania tomentosa (Benth.) Fritsch (Oiti), seguida de Moraceae, com 19 indivíduos pertencentes a duas espécies. A família Fabaceae destacou-se com seis espécies, seguida por Arecaeae, Malvaceae e Moraceae, com duas espécies cada, as demais famílias foram representadas por apenas uma espécie (Tabela 1).

Do total de sete praças distribuídas pela cidade, a Praça Maria Alves não apresenta vegetação e a Praça José Bonifácio de Andradas possui apenas indivíduos jovens, por isso, as mesmas não foram inventariadas. Na Praça José Augusto de Oliveira, foi encontrado apenas um exemplar da espécie $C$. glabrata, espécie nativa da região; semelhante ao observado na Praça do Locomóvel, onde foi encontrado apenas um indivíduo de P. guajava. A Praça Coronel Daniel da Fonseca, apresentou 13 indivíduos, distribuídos em cinco gêneros, quatro famílias e cinco espécies, sendo elas, L. tomentosa, D. regia, Bauhinia sp., F. benjamina e Plumeria sp. Na Praça Miguel da Malária, foram encontrados seis indivíduos, distribuídos em duas famílias e três espécies (C. grandis, L. tomentosa e $D$. regia). A Praça Cristo Redentor, que é situada na região central da cidade, apresentou 28 indivíduos, distribuídos em oito famílias. A espécie que apresentou maior frequência foi $L$. tomentosa, com 11 exemplares, as espécies $C$. revoluta, $D$. regia, $P$. pluviosa, $C$. speciosa e $G$. americana, foram representadas por apenas um indivíduo de cada espécie.

A arborização com o uso de espécies de porte adequado para calçadas, praças e parques contribui com a diminuição das alterações climáticas em pequena escala (ABREUHARBICH; LABAKI; MATZARAKIS, 2015; AGUIRRE JUNIOR; LIMA, 2007), além de atribuir beleza ao ambiente proporcionando prazer estético e bem-estar psicológico (CEMIG, 2011), características desejadas para os ambientes citados. 
Tabela 1. Espécies, frequência e atributos ecológicos que compõem a arborização urbana das praças e avenidas de Jequitaí, MG

Table 1. Species, frequency and ecological attributes that compose an urban afforestation of the squares and avenues of Jequitaí, MG

\begin{tabular}{|c|c|c|c|c|c|c|c|c|}
\hline Família / Nome científico & Nome popular & Origem & FA & $\begin{array}{l}\text { FR } \\
(\%)\end{array}$ & $\begin{array}{l}\text { DC } \\
(\%)\end{array}$ & $\begin{array}{l}\text { CR } \\
(\%)\end{array}$ & SP & SD \\
\hline \multicolumn{9}{|l|}{ Annonaceae } \\
\hline $\begin{array}{l}\text { Annona squamosa L. } \\
\text { Apocynaceae }\end{array}$ & \multicolumn{7}{|c|}{ Apocynaceae } & Zooc. \\
\hline $\begin{array}{l}\text { Plumeria sp. } \\
\text { Arecaceae }\end{array}$ & Jasmim-manga & Exótica/Cultivada & 1 & 0,9 & 0 & 0 & Esf./Fal & Anec. \\
\hline $\begin{array}{l}\text { Dypsis lutescens (H. Wendl.) Beentje } \\
\text { \& J. Dransf. }\end{array}$ & $\begin{array}{l}\text { Palmeira-areca- } \\
\text { bambu }\end{array}$ & Exótica/Cultivada & 1 & 0,9 & 0 & 100 & Ent. & Zooc. \\
\hline $\begin{array}{l}\text { Roystonea oleraceae (Jacq.) O.F. } \\
\text { Cook } \\
\text { Bignoniaceae }\end{array}$ & Palmeira-imperial & Exótica/Cultivada & 5 & 4,5 & 0 & 0 & Ent. & Zooc. \\
\hline \multicolumn{9}{|l|}{ Boraginaceae } \\
\hline $\begin{array}{l}\text { Cordia glabrata (Mart.) A.DC. } \\
\text { Chrysobalanaceae }\end{array}$ & Caraiba & Nativa/Região & 1 & 0,9 & 0 & 0 & Ent. & Zooc. \\
\hline $\begin{array}{l}\text { Licania tomentosa (Benth.) Fritsch } \\
\text { Combretaceae }\end{array}$ & \multicolumn{8}{|c|}{ Combretaceae } \\
\hline $\begin{array}{l}\text { Terminalia catappa L. } \\
\text { Cupressaceae }\end{array}$ & Sete-copas & Exótica/Naturalizada & 11 & 9,9 & 64 & 55 & Mel. & Zooc. \\
\hline \multicolumn{9}{|l|}{ Cycadaceae } \\
\hline $\begin{array}{l}\text { Cycas revoluta Thunb. } \\
\text { Euphorbiaceae }\end{array}$ & \multicolumn{7}{|c|}{ Euphorbiaceae } & Auto. \\
\hline \multicolumn{9}{|l|}{ Fabaceae } \\
\hline Bauhinia L. & Pata-de-vaca & & 1 & 0,9 & 0 & 0 & Mel. & Aut. \\
\hline $\begin{array}{l}\text { Poincianella pluviosa (DC.) } \\
\text { L.P.Queiroz }\end{array}$ & Sibipiruna & Nativa/Região & 3 & 2,7 & 67 & 67 & Mel. & Aut. \\
\hline Cassia grandis L.f. & Cassia-rosa & Nativa/Região & 1 & 0,9 & 0 & 100 & Mel. & Aut. \\
\hline Delonix regia (Bojer ex Hook.) Raf. & Flamboyant & Exótica/Cultivada & 3 & 2,7 & 0 & 33 & Mel. & Aut. \\
\hline Caesalpinia pulcherrima (L.) Sw. & Flamboyant-mirim & Exótica/Naturalizada & 1 & 0,9 & 0 & 0 & Orn. & Aut. \\
\hline \multicolumn{9}{|l|}{ Malvaceae } \\
\hline Ceiba speciosa (A.St.-Hil.) Ravenna & Barriguda & Nativa/Região & 1 & 0,9 & 0 & 0 & Orn & Anec. \\
\hline $\begin{array}{l}\text { Pachira glabra Pasq. } \\
\text { Moraceae }\end{array}$ & $\begin{array}{l}\text { Castanha-do- } \\
\text { maranhão }\end{array}$ & Exótica/Naturalizada & 2 & 1,8 & 0 & 0 & Quir. & Aut. \\
\hline Ficus benjamina L. & Figueira & Exótica/Naturalizada & 18 & 16,2 & 56 & 28 & Mel. & Zooc. \\
\hline \multicolumn{9}{|l|}{ Myrtaceae } \\
\hline \multicolumn{9}{|l|}{ Rubiaceae } \\
\hline $\begin{array}{l}\text { Genipa americana L. } \\
\text { Rutaceae }\end{array}$ & \multicolumn{7}{|c|}{ Rutaceae } & I Zooc \\
\hline Murraya paniculata (L.) Jack & Murta & Exótica/Cultivada & 12 & 10,8 & & 0 & Ent. & Zooc. \\
\hline
\end{tabular}

Nota: Frequência Absoluta (FA); Frequência Relativa (FR); Conflito com calçada (DC); Conflito rede aérea de energia elétrica (CR); Síndrome de Polinização (SP); Cantarofilia (Cant.); Melitofilia (Mel.); Entomofilia (Ent.); Anemofilia (Anef.); Ornitofilia (Orn.); Quiropterofilia (Quir.); Esfingofilia (Esf.); Falenofilia (Fal.). Síndrome de dispersão (SD); Autocoria (Aut.); Zoocoria (Zooc.); Anemocoria (Anec.). * Gimnospermas polinizadas pelo vento 
$\mathrm{Na}$ Avenida Espírito Santo, foram observados seis indivíduos de seis famílias e seis espécies (A. squamosa, C. revoluta, T. stans, P. guajava, L. tomentosa e T. catappa). A Avenida Estados Unidos apresenta 30 indivíduos de sete famílias e sete espécies, T. catappa apresenta a maior frequência, com 10 indivíduos, seguida pela $L$. tomentosa, nove indivíduos. As espécies P. glabra e P. guajava ocorrem em menor frequência, com um indivíduo cada. A Avenida Sete de Setembro apresenta 15 indivíduos, distribuídos em cinco famílias, seis gêneros e sete espécies. F. benjamina é a espécie que apresenta maior frequência, com 10 indivíduos. A Avenida dos Andradas apresenta 11 indivíduos distribuídos em cinco famílias, cinco gêneros e cinco espécies. M. paniculata apresentou maior frequência, com cinco indivíduos; as espécies $D$. lutescens, $R$. communis e $P$. glabra, estão representadas por apenas um indivíduo cada.

Com relação à frequência, as espécies $L$. tomentosa (com 33,33\% dos indivíduos), $F$. benjamina (com 16,22\% dos indivíduos) e M. paniculata (com 10,81\% dos indivíduos), estão acima do recomendado pela Cemig (2011), que sugere que a densidade não ultrapasse $10 \%$ de uma mesma espécie. Essas espécies representam as maiores frequências absolutas e relativas da comunidade (Tabela 1). L. tomentosa apresentou indivíduos distribuídos em diferentes ambientes analisados. Essa espécie também foi a mais frequente nas cidades de Alta Floresta, Carlinda e Nova Monte Verde no estado do Mato Grosso (ALMEIDA; RONDON NETO, 2010).

Poincianella pluviosa, está representada por apenas três indivíduos, é uma espécie nativa da região que apresenta potencial para ser plantada em locais apropriados. É a terceira mais abundante em Montes Claros, cidade com condições climáticas similares a Jequitaí (VELOSO et al. 2015). Segundo Abreu-Harbich, Labaki e Matzarakis (2015), essa espécie se destacou em função da capacidade de atenuar a temperatura e promover a melhoria do conforto térmico, reduzindo a temperatura entre 12 e $16^{\circ} \mathrm{C}$ em conjunto de árvores e individualmente entre 12,5 e $14,5^{\circ} \mathrm{C}$.

Com a análise da altura, constatou-se que 55\% dos indivíduos encontrados na arborização dos ambientes analisados apresentam pequeno porte, com altura abaixo de $6 \mathrm{~m}$, dentre eles se destacam as espécies C. pulcherrima, M. paniculata, T. stans e C. revoluta, $44 \%$ com porte médio, entre 6 e $10 \mathrm{~m}$, destacando F. benjamina, D. regia, L. tomentosa, F. benjamina e C. glabrata e 12 indivíduos apresentam altura superior a $10 \mathrm{~m}$, representados principalmente por T. catappa, L. tomentosa, C. grandis e H. courbaril. A altura de alguns indivíduos analisados foi afetada pela poda. A prática de poda é utilizada normalmente para solucionar conflitos, entre partes da árvore com a rede elétrica, iluminação pública, a sinalização de trânsito ou mesmo com as fachadas de edificações, impedindo o contato entre estes componentes e a vegetação (CEMIG, 2011). No entanto, a arborização de grande porte tem se tornado diferencial nas cidades com relação à qualidade de vida (AGUIRRE JUNIOR; LIMA, 2007). 
Com os dados de diâmetros mensurados, verificou-se que $36 \%$ dos indivíduos apresentam DAP menor que $30 \mathrm{~cm}$, destacando as espécies M. paniculata, $P$. guajava, $T$. stans, $P$. glabra, 58\% estão entre 30 e $60 \mathrm{~cm}$, sendo representados principalmente por L. tomentosa, T. catappa, C. glabrata, L. tomentosa e C. grandis e $5 \%$ entre 60 e $80 \mathrm{~cm}$, representados por $D$. regia, L. tomentosa e $H$. courbaril e apenas $2 \%$, que é representado pela $L$. tomentosa, apresenta o DAP superior a $80 \mathrm{~cm}$. A maior concentração de indivíduos entre 30 e $60 \mathrm{~cm}$ indicam que essa população não é jovem, e provém de plantios antigos ou são remanescentes das vegetações nativas do local.

Através do diagnóstico da arborização urbana, averiguou-se que 26 indivíduos apresentam problemas com rede aérea de energia elétrica, destes destacam-se as espécies $L$. tomentosa (10 indivíduos), T. catappa (seis indivíduos) e F. benjamina (cinco indivíduos). Trinta indivíduos de seis espécies apresentam conflitos com a pavimentação, destacando $F$. benjamina (10 indivíduos), L. tomentosa (nove indivíduos) e T. catappa (sete indivíduos), causando rachaduras. Dessa forma, $50 \%$ dos indivíduos apresentam algum tipo de conflito, com a rede aérea de energia elétrica e/ou pavimentação. Destacam-se os indivíduos das espécies $T$. catappa, P. pluviosa e F. benjamina, em que 64\%, 67\% e 56\%, respectivamente, apresentam problemas com a calçada e 55\% dos indivíduos de $T$. catappa e $67 \%$ dos indivíduos de $P$. pluviosa apresentam problemas com a rede aérea de energia elétrica.

Para as espécies que estão em conflito com a rede aérea de energia elétrica, observouse que o local é inadequado para seu desenvolvimento, que receberam manejo inadequado ou que rede aérea de energia elétrica é inadequada para a implementação da arborização urbana. Sob a rede aérea de energia elétrica, deve-se preferir espécies de menor porte, que ofereçam a possibilidade de condução da copa e crescimento lento. Deve-se levar em conta porte da planta, o local onde vai ser plantada, pois se não houver um planejamento adequado, problemas de conflitos podem ocorrer. Uma alternativa para reduzir conflitos, é a distribuição da energia elétrica subterrânea, este tipo de rede evita conflitos com as copas das árvores, mas está sujeita a conflito com raízes (CEMIG, 2011).

A poda foi verificada em $59 \%$ dos indivíduos, sendo que apenas um indivíduo apresentou poda drástica. No trabalho realizado por Santos et al. (2018), 25,5\% dos indivíduos apresentaram conflito com a calçada, 3,5\% conflito com rede aérea de energia elétrica e 12,6\% apresentaram sinais de podas drásticas.

Com relação ao estado geral, 75\% indivíduos apresentaram boa condição (condição média de vigor, necessitando de reparos ou podas), 10\% estavam em ótima condição, 14\% em condição regular (descaracterização da arquitetura, injúria mecânica, requer reparo) e 2\% indivíduos estavam em condição péssima (declínio irreversível, ataque muito severo por insetos). De acordo com Costa et al. (2017), o estado de conservação da vegetação urbana, é de grande 
importância, pois reflete no embelezamento dos ambientes onde estão inseridas e contribuem para estarem sempre atraentes e serem frequentados pela população.

Em relação à origem das espécies, $37 \%$ são nativas, sendo $29 \%$ são nativas da região e $8 \%$ nativas de outras regiões do Brasil, enquanto $63 \%$ são exóticas. A frequência dos indivíduos de espécies nativas de outras regiões do Brasil e de exóticas naturalizadas é similar, representando $34 \%$ dos indivíduos cada, $24 \%$ dos indivíduos pertencem a espécies exóticas cultivadas e $8 \%$ a espécies nativas da região. A utilização de espécies exóticas não deve ser eliminada em ambientes urbanos, essas espécies podem ser empregadas a fim de complementar as espécies nativas neste ambiente (SJÖMAN et al., 2016).

Das espécies que compõem a arborização de Jequitaí, algumas exóticas como Roystonea oleraceae, Tecoma stans, Caesalpinia pulcherrima, Pachira glabra e Murraya paniculata, destacaram-se, pois não apresentam conflitos com elementos estruturais existentes no meio urbano e interagem com a fauna polinizadora ou dispersora. Espécies nativas da região como Cordia glabrata, Hymenaea courbaril e Genipa americana, devem ser estimuladas na arborização de praças, pois podem atingir grande porte e interagem com a fauna e apresentam um bom estado de conservação. As espécies nativas da região com frutos carnosos devem ser priorizadas na arborização, pois mantêm as interações da flora e fauna local, auxiliando para manter o equilíbrio e a funcionalidade do ecossistema urbano.

A polinização pelo vento foi observada em $13 \%$ das espécies, destacando-se as Gimnospermas. Em relação às polinizações bióticas, a cantarofilia ocorreu em $4 \%$ das espécies, a entomofilia em $17 \%$ e a melitofilia em $42 \%$, enquanto a esfingofilia/falenofilia, ornitofilia e quiropterofilia em $8 \%$ cada. A anemofilia foi notada em $4 \%$ dos indivíduos, cantarofilia em $1 \%$, entomofilia em $17 \%$, esfingofilia/falenofilia em $2 \%$, melitofilia em $71 \%$, ornitofilia em $2 \%$ e a quiropterofilia em $3 \%$ dos indivíduos. A melitofilia se configura como a síndrome de polinização mais frequente entre as espécies e indivíduos analisados, sendo também a mais comum entre as espécies nativas da região e exóticas (Figura 3). Essa síndrome de polinização é tipicamente predominante em ecossistemas naturais (ARAÚJO et al., 2009). 


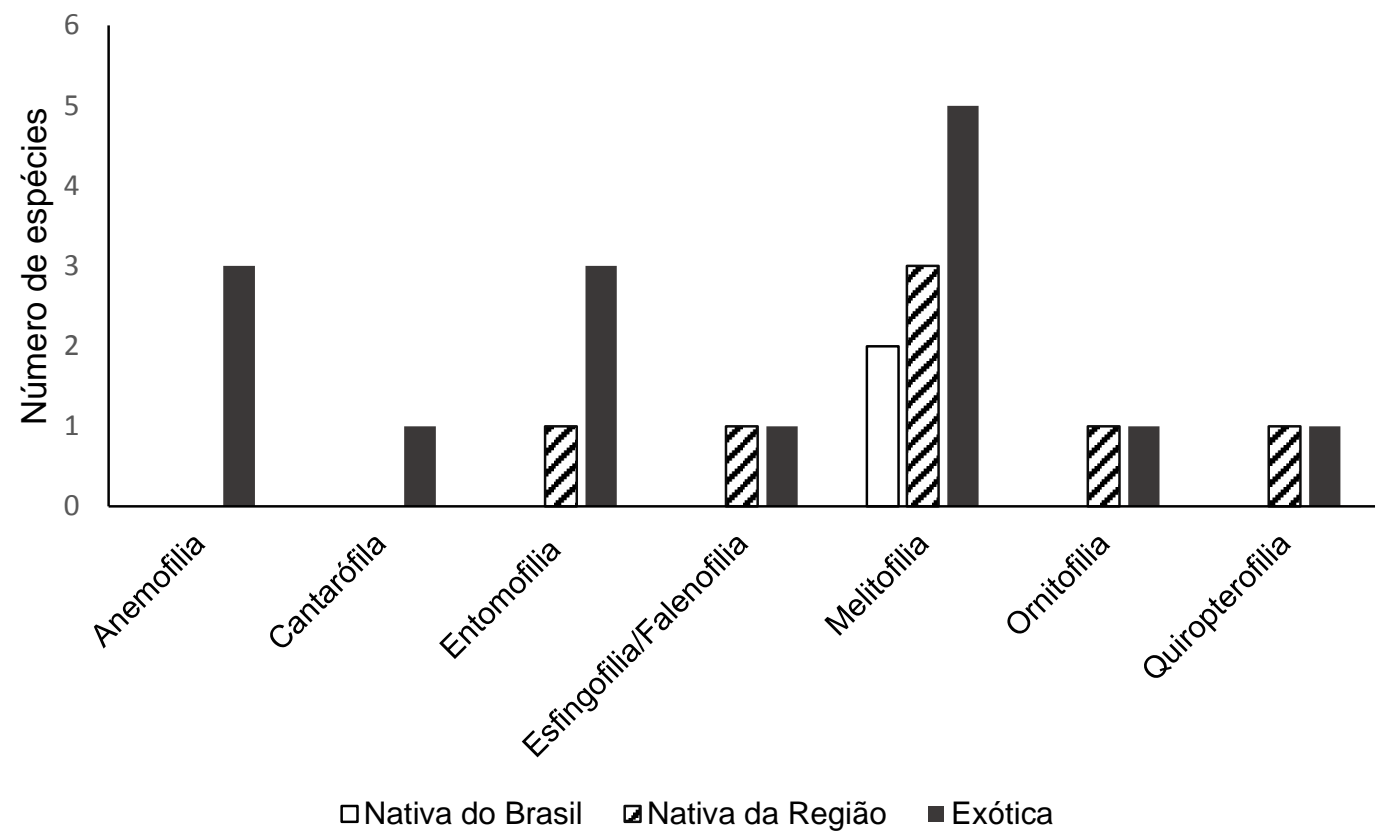

Figura 3. Síndrome de polinização em relação à origem das espécies analisadas, nas praças e avenidas de Jequitaí, MG

Figure 3. Pollination Syndromes in relation to the origin of the analyzed species, in the squares and avenues of Jequitaí, MG

A principal síndrome de dispersão foi a zoocoria (50\% das espécies, $84 \%$ dos indivíduos), seguida pela autocorica (33\% das espécies, 13\% dos indivíduos) e anemocórica (17\% das espécies, $4 \%$ dos indivíduos). A zoocoria e autocoria foram as mais frequentes nas espécies exóticas e nas nativas da região (Figura 4).

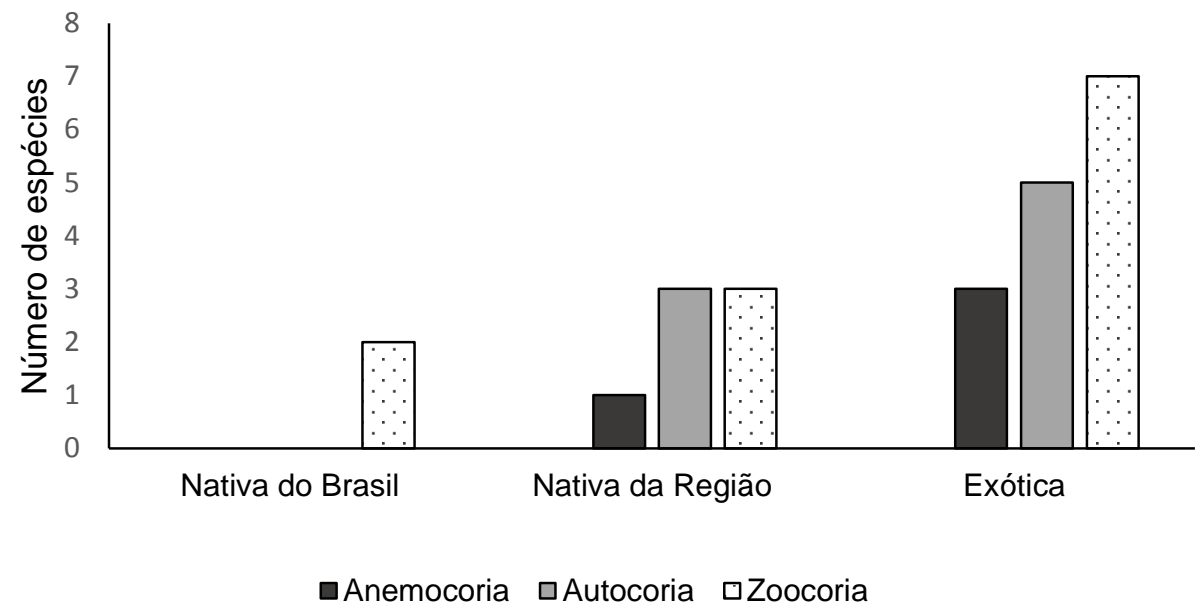

Figura 4. Síndromes de dispersão em relação à origem das espécies analisadas nas praças e avenidas de Jequitaí, MG

Figure 4. Dispersal Syndromes in relation to the origin of the species analyzed in the squares and avenues of Jequitaí, MG 
Dentre os frutos zoocóricos, a maior parte das espécies possui frutos pequenos, coloridos e atrativos para a avifauna ou para morcegos. Estes grupos de animais são de grande interesse em ambientes urbanos, pois são importantes agentes polinizadores e dispersores de sementes para outras áreas e contribuem efetivamente na restauração de ecossistemas degradados (CAMPOS et al., 2012). Além disso, a presença de avifauna é um indicador de qualidade ambiental dos centros urbanos (MATOS; QUEIROZ, 2009).

O índice de Shannon foi de 2,32, este resultado está abaixo da média de outros estudos e próximo ao levantamento em Montes Claros (Tabela 2). O estudo com diversidade inferior foi o de Santos et al. (2013), realizado no o município de Lages, SC. No entanto, segundo o autor, o resultado se deve à elevada porcentagem da espécie Ligustrum lucidum e ao baixo número de espécies da arborização. Para o índice de equabilidade, no presente estudo foi calculado o valor de 0,096, considerado baixo quando comparado com Santos et al. (2013). As métricas ecológicas de diversidade e, principalmente, equabilidade, são pouco usuais em estudos de arborização urbana, apesar de serem de grande utilidade para a interpretação da situação do ecossistema urbano.

Tabela 2. Índice de Shannon ( $\left.H^{\prime}\right)$ e equabilidade $(\mathrm{J}$ ') deste trabalho e de outros Table 2. Shannon index $\left(H^{\prime}\right)$ and equability $\left(J^{\prime}\right)$ of this study and others

\begin{tabular}{lllll}
\hline Autor & Local & Número de indivíduos & H'$^{\prime}$ & J' \\
\hline Da autora (2019) & - & 111 & 2,32 & 0,096 \\
Costa et al. (2017) & Caxias, MA & 263 & 2,75 & - \\
Santos et al. (2013) & Lages, SC & 149 & 1,25 & 0,49 \\
Veloso et al. (2015) & Montes Claros, MG & 1605 & 2,38 & - \\
\hline
\end{tabular}

\section{CONCLUSÃO}

A arborização da cidade de Jequitaí é composta, em sua maioria, por espécies exóticas, no entanto há maior frequência de indivíduos pertencentes a espécies nativas de outras regiões do Brasil ou exóticas naturalizadas, culminando em uma comunidade vegetal de baixa diversidade. As espécies nativas da região, como Cordia glabrata, Hymenaea courbaril e Genipa americana, se mostram adaptadas ao ambiente urbano, interagem com a fauna e apresentam um bom estado de conservação, sendo sugeridas para o plantio em praças.

A polinização por melitofilia e a dispersão por zoocoria foram as estratégias mais frequentes entre as espécies e indivíduos. Algumas espécies nativas apresentaram grande potencial para ambientes urbanos, devendo ser priorizadas pois contribuem para a manutenção da conservação da flora e fauna local, além do equilíbrio no ecossistema urbano. 


\section{REFERÊNCIAS}

ABREU-HARBICH, L. V. DE; LABAKI, L. C.; MATZARAKIS, A. Effect of tree planting design and tree species on human thermal comfort in the tropics. Landscape and Urban Planning, Amsterdam, v. 138, p. 99-109, 2015.

AGUIRRE JUNIOR, J. H. DE; LIMA, A. M. L. P. Uso de árvores e arbustos em cidades brasileiras. Revista da Sociedade Brasileira de Arborização Urbana, Piracicaba, v.2, n.4, p. 50-66, 2007.

ALMEIDA, D. N.; RONDON NETO, R. M. Análise da arborização urbana de três cidades da região norte do Estado de Mato Grosso. Acta Amazonica. Manaus, v. 40, n. 4, p. 647 - 656, 2010.

ARAÚJO, J. L. O.; QUIRINO, Z. G. M.; NETO, P. C. G.; ARAÚJO, A. C. Síndromes de polinização ocorrentes em uma área de Mata Atlântica, Paraíba, Brasil. Revista Biotemas, Florianópolis, v. 22, n. 4, p. 83-94, 2009.

BENINI, R. DE M.; MENDIONDO, E. M. Urbanização e Impactos no Ciclo Hidrológico na Bacia do Mineirinho. Floresta e Ambiente, Seropédica, v. 22. N. 2, p. 211-222, 2015.

BRUN, F. G. K.; LINK, D.; BRUN, E. J. O emprego da arborização na manutenção da biodiversidade de fauna em áreas urbanas. Revista Da Sociedade Brasileira De Arborização Urbana, Piracicaba, v. 2, n. 1, p. 117-127, 2007.

CADORIN, D. A.; MELLO, N. A. DE. Efeitos da impermeabilização dos solos sobre a arborização no município de Pato Branco-PR. Synergismus scyentifica, Pato Branco, v. 6, n.1, p. 1-8, 2011.

CAMPOS, W. H.; NETO, A. M.; PEIXOTO, H. J. C.; GODINHO, L. B.; SILVA, E. Contribuição da fauna silvestre em projetos de restauração ecológica no Brasil. Pesquisa Florestal Brasileira, Colombo, v. 32, n. 72, p. 429-440, 2012.

CEMIG Companhia Energética de Minas Gerais. Manual de arborização. Belo Horizonte: Cemig / Fundação Biodiversitas, 2011. 112 p.: ilust.

COSTA, C. F.; FONSECA, R. S.; ALMEIDA, D. B. de; OLIVEIRA, M. S. de; OLIVEIRA, D. S; BRAGA, J. H. P. Espécies utilizadas na arborização em praças do município de Caxias, Maranhão. Revista da Sociedade Brasileira de Arborização Urbana, Piracicaba, v.12, n.1, p. 65-78, 2017.

COSTA, D. F. DA; PERES, L. DE F.; SILVA, H. R. Identificação de ilhas de calor na área urbana de Ilha Solteira-SP através da utilização de geotecnologias. In.: XIV Simpósio Brasileiro de Sensoriamento Remoto, 2009, Anais... Natal, Brasil, 25-30 abril INPE, p. 647-653.

FAEGRI, K.; PIJL, L. The principles of pollination ecology. Oxford, Pergamon Press. 1976.

Flora do Brasil 2020 em construção. Jardim Botânico do Rio de Janeiro. Disponível em: <http://floradobrasil.jbrj.gov.br/ >. Acesso em: 22 mai 2019.

GÓMEZ-BAGGETHUN, E.; BARTON, D. N. Classifying and valuing ecosystem services for urban planning. Ecological Economics, Amsterdam, v. 86. P. 235-245, 2013. 
IBGE (Instituto Brasileiro de Geografia e Estatística). Brasil. Minas Gerais. Jequitaí. 2017. Disponível em: <https://cidades.ibge.gov.br/v4/brasil/mg/jequitai/panorama>. Acesso em: 22 mai 2019.

MAGURRAN, A. E. Medindo a diversidade biológica. Curitiba: Editora UFPR, 261 p. 2011.

MATOS, E.; QUEIROZ, L. P. Árvores para cidades. Salvador: Ministério Público do Estado da Bahia: Solisluna, 2009. 340 p.: il.

PASTORE, M.; RODRIGUES, R. S.; SIMÃO-BIANCHINI, R.; FILGUEIRAS, T. de S. Plantas exóticas invasoras na reserva biológica do alto da serra de Paranapiacaba, Santo André SP: guia de campo. São Paulo: Instituto de Botânica, 2012. 293p.

SANTOS, É. M.; SILVEIRA, B. D.; SOUZA, A. C.; SCHMITZ, V.; SILVA, A. C.; HIGUCHI, P. Análise quali-quantitativa da arborização urbana em Lages, SC. Revista de Ciências Agroveterinárias, Lages, v.12, n.1, p. 59-67, 2013.

SANTOS, R. C. dos; BESSEGATTO, D.; ANTUNES, L.; MALENGO, F. de M.; Análise qualiquantitativa da arborização urbana do centro da cidade de Sananduva-RS. Revista gestão sustetabilidade ambiental, Florianópolis, v. 7, n. 2, p. 143-158, 2018.

SILVA FILHO, D. F.; PIZETTA, P. U. C.; ALMEIDA, J. B. S. A.; PIVETTA, K. F. L.; FERRAUDO, A. S. Banco de dados relacional para cadastro, avaliação e manejo da arborização em vias públicas. Revista Árvore, Viçosa, v.26, n.5, p.629-642, 2002.

SJÖMAN, H.; MORGENROTH, J.; SJÖMAN, J. D.; SÆBØ, A.; KOWARIKE, I. Diversification of the urban forest-Can we afford to exclude exotic tree species? Urban Forestry \& Urban Greening, Amsterdam, v. 18, n. 1, p. 237-241, 2016.

UNITED NATIONS, DEPARTMENT OF ECONOMIC AND SOCIAL AFFAIRS, POPULATION DIVISION. World Urbanization Prospects: The 2014 Revision. New York: United Nations, 2015. 493p.

VAN DER PIJL, L. Principles of dispersal in higher plants. Berlim: Springer-Verlag, 1982, $215 p$.

VELOSO, M. D. M.; BRAGA, L. L.; RODRIGUES, P. M. S.; MIRANDA, W. O.; BRANDAO, D. O.; NUNES, Y. R. F. Caracterização da arborização urbana em diferentes ambientes na cidade de Montes Claros, MG, Brasil. Revista da Sociedade Brasileira de Arborização Urbana, Piracicaba, v. 9, p.118-133, 2015. 